\title{
A load distribution theory for bridge slabs allowing for the effect of Poisson's ratio*
}

\author{
by R. E. Rowe, B.A.
}

\section{Contribution by J. A. N. Lee $\dagger$}

The contributor has had some difficulty in understanding the following statement on page 70 :

"The solution to equation (a) is

$$
w_{m}=\frac{16 a^{4}}{m^{4} \pi^{4} D} \frac{H_{m}}{2 b} \sin m \alpha
$$

which satisfies the conditions at the supports and free edges."

Perhaps Mr Rowe would explain this statement a little more, since this solution does not seem to satisfy the equation for bending moment in the $y$ direction as given in equation (3) on page 71 .

Although it is realized that it is convenient to provide a solution which is a mere amendment to Guyon's results, it is felt that whereas Guyon's mean deflexion suited the then specified boundary conditions, this partial deflexion is not a true result, since it does not satisfy the prevailing conditions. Moreover, it is not necessary at this stage in the calculations to say whether it fits the boundary conditions, since it is only necessary for the completed solution to fulfil these stipulations.

Furthermore, would the author elucidate as to the algebraic value of mean deflexion to be used for the case of a point load anywhere on the surface of a slab?

\section{Contribution by J. B. Walton*}

It is interesting to observe from Mr Rowe's paper that, when calculating the magnitude of the longitudinal bending moments in a prestressed concrete bridge slab, no account need be taken of lateral strain in the slab, and that generally the effect of Poisson's ratio in such a slab is only appreciable at the edges for eccentric loading.

Such investigations are of particular interest to the designer in so far as they substantiate the normal practice of designing for longitudinal bending moments. Furthermore, when an abnormal load is to cross a particular bridge, prior notice is generally received by the responsible bridge authority, thereby enabling arrangements to be made for the heavy load to be directed along such a path over the bridge that the more serious effects of eccentric loading are avoided.

If allowance for the effect of Poisson's ratio is made in the calculations for the transverse strength of a prestressed concrete bridge slab, then it would appear that design standards should be such that cracks (other than microscopic cracks-see the remarks of Dr Abeles\$) should be avoided.

On the basis of this limitation it is suggested that the longitudinal prestressing force should be such that, under any condition of loading, the extreme bottom fibre stress in the deck slab nowhere exceeds one-third of the stress which would cause the concrete to crack.

It appears unnecessary to make a similar design stress stipulation when dealing with the transverse prestressing force, since difficulty does not usually arise in arranging lateral prestressing reinforcement so that it does not produce tensile stress in the concrete.

\section{Contribution by $Y$. Guyon}

Mr Rowe's study is certainly very useful and shows that appreciable errors may arise although still in the elastic zone-due to ignoring Poisson's ratio.

(1) Concerning the coefficient $K$ (distribution coefficient for longitudinal moments), the errors are not very serious and the comparison of Table 1 (page 74) with my own results for $\sigma=0$ does not indicate errors greater than $30 \%$; the greatest errors appear at the edges.

Concerning this coefficient $K$, I must correct an error which I have made. The ratio of the deflexion at some longitudinal section, $w$, to the average deflexion $W$ is

$$
\frac{w}{W}=\frac{K_{1} W_{1}+K_{2} W_{2}+\ldots}{W_{1}+W_{2}+\ldots}
$$

Similarly, if $m$ and $M$ are the actual moment at some longitudinal section and the average moment, we have:

$$
\frac{m}{M}=\frac{K_{1} M_{1}+K_{2} M_{2}+\ldots}{M_{1}+M_{2}+\ldots}
$$

I have suggested that we may limit ourselves to the

\footnotetext{
*Pages 69-78.

†Departments of Civil and Mechanical Engineering, University of Nottingham.

¥City Engineer's Department, Sheffield.

§HOLLAND, A. D. Prestressed units for short-span highway bridges. Proceedings of the Institution of Civil Engineers. Part II, Vol. 4, No. 2.

June 1955. Contribution to the discussion by ABELES, P. W. pp. 264-269.
} 
first terms of the series, taking simply $\frac{w}{W}=K_{1} ; \frac{m}{M}=K_{1}$.

This is perfectly exact for the deflexion $w$. It may, however, be inexact for the moment if the loads are very concentrated in the spanwise direction (i.e. distributed over a very short length of the span). In this case it is better to take two or three terms of the series, which may be done with the tables and graphs I have given.

(2) As Mr Rowe very rightly says, the errors which arise due to neglecting Poisson's ratio are especially important in the case of the transverse moment; they may reach $40 \%$. It is thus advisable to use the values which he has found when designing slabs in the elastic zone.

(3) I would like to ask the following question. Experiment shows that one can use slabs elastically under loads appreciably greater than those given by the elastic theories. This is only possible thanks to a redistribution of the moments due to micro-cracking, quite invisible, and which in my opinion does not cause any deterioration in the strength of the slab.

Must we under these conditions still worry about Poisson's ratio?

This raises, in fact, the whole question of knowing whether we should design slabs elastically or on the ultimate load theory with reasonable factors of safety.

As the experimental evidence accumulates, I incline more and more to the latter.

\section{Reply by the author}

As Mr Lee points out, the solution to part (a) of the governing differential equation does not satisfy the boundary conditions at the free edges. The statement I made regarding this point was too sweeping at that particular stage of the analysis. It is only later when the complete expression for the partial deflexion is obtained, i.e. the sum of the solutions to parts (a) and (b) of the governing differential equation, that the boundary conditions at the free edges can be satisfied.

For a point load anywhere on the surface of the slab, the mean deflexion is obtained by considering the slab as a simply supported beam subjected to a uniformly distributed line load through the point of application of the load, the intensity of loading being the value of the point load divided by the width of the slab.

$\mathrm{Mr}$ Walton raised the point that if a bridge slab is designed to prevent cracking in the transverse direction, due allowance being made for the Poisson's ratio effect, then it would seem reasonable to allow some tensile stress in the extreme longitudinal fibres. This point is of particular interest for, if some longitudinal tensile stress were allowed under the Ministry of Transport abnormal loading, considerable economies in materials would result. For the quality of concrete used in prestressed designs, a tensile stress of the magnitude of 200-300 $\mathrm{lb} / \mathrm{in}^{2}$ would seem reasonable.

M. Guyon raised the point that when considering longitudinal moments it is inexact to consider only the first term of the infinite series for loads distributed over a short length of the span. Experiments ${ }^{(7)}$ have shown that this is so, but that for most practical forms of loading the longitudinal moments under the loads are given with sufficient accuracy by the first term only, provided the moments so found are increased by $10 \%$. More terms could be considered if required, as M. Guyon says, but such further complication is unnecessary.

M. Guyon's final point poses the question of elastic versus ultimate load theory as a basis for design. It is well known that slabs do possess a great reserve of strength and remain apparently elastic at loads in excess of those given by the elastic theories. Under such conditions with micro-cracking present, I would say that Poisson's ratio would still have some effect on the interaction of the principal moments, though to what extent I don't know.

The analysis given in the paper is of particular use in pseudo-slab bridges, i.e. bridges of a number of beams jointed together and stressed transversely. In this case there is no tensile strength transversely apart from that provided by the transverse stress and hence it is of paramount importance that the transverse moments be determined accurately so that the correct amount of stress can be applied.

Should ultimate load theory be used as a basis for bridge design, then an elastic analysis would probably have to be used to check the stresses under working load conditions and Poisson's ratio would still be important. 